\title{
Smoking-induced changes in cancer-related factors in patients with upper tract urothelial cancer
}

\author{
YASUYOSHI MIYATA, KENSUKE MITSUNARI, ASAI AKIHIRO, \\ SHIN-ICHI WATANABE, YASUSHI MOCHIZUKI and HIDEKI SAKAI
}

\begin{abstract}
Department of Urology, Nagasaki University Graduate School of Biomedical Sciences, Nagasaki 852-8501, Japan
\end{abstract}
Received August 4, 2014; Accepted November 14, 2014

DOI: $10.3892 / \mathrm{mco} .2014 .471$

\begin{abstract}
Cigarette smoking is a major risk factor for urothelial cancer (UC) development. However, the associations between smoking and changes in the pathological characteristics and molecular expression of cancer-related molecules in upper tract (UT) UC have not been fully elucidated. We investigated the associations between smoking status and cancer-related factors, including cancer cell proliferation, apoptosis, angiogenesis, lymphangiogenesis and expression of vascular endothelial growth factor-A and $-\mathrm{C}$, matrix metalloproteinase (MMP)-2 and -9, cyclooxygenase (COX)-2 and urokinase-type plasminogen activator, in patients with UTUC. A total of 134 patients who underwent nephroureterectomy were retrospectively investigated. Proliferation index (PI), microvessel density and lymphatic vessel density (LVD) were measured using anti-Ki-67, anti-CD105 and anti-D2-40 antibodies in formalin-fixed specimens. The apoptotic index was evaluated using the terminal deoxynucleotidyl transferase-mediated dUTP nick end labeling method. Other cancer-related molecules were investigated by immunohistochemistry in similar specimens. The patients were divided into three groups; non-smoker $(n=54,40.3 \%)$, former smoker $(n=46,34.3 \%)$ and current smoker $(\mathrm{n}=34,25.4 \%)$. The PI and the apoptotic index were not found to be correlated with smoking status; however, the mean/standard deviation level of LVD in current smokers (40.9/12.9) was significantly higher $(\mathrm{P}=0.034)$ compared to that in patients who had never smoked (34.4/10.6). In addition, smoking status was positively correlated with the presence of intratumoral lymphatic vessels (iLV) $(\mathrm{P}=0.010)$ and the expression of COX-2 and MMP-9 ( $\mathrm{P}=0.032)$. The multivariate analysis demonstrated that current smoking was independently associated with all the abovementioned smoking-related factors. However, former smoking was correlated with LVD and the presence of iLV. In the survival analysis, LVD, the
\end{abstract}

Correspondence to: Dr Yasuyoshi Miyata, Department of Urology, Nagasaki University Graduate School of Biomedical Sciences, 1-7-1 Sakamoto Street, Nagasaki 852-8501, Japan

E-mail: int.doc.miya@m3.dion.ne.jp

Key words: smoking, upper tract urothelial cancer, prognosis, lymphangiogenesis, cyclooxygenase-2, matrix metalloproteinases presence of $\mathrm{iLV}$ and the expression of COX-2 and MMP-9 were identified as predictive factors for metastasis following surgery. In conclusion, lymphangiogenesis and the expression levels of COX-2 and MMP-9 were found to be associated with the smoking status of UTUC patients. Our results may provide important insights into the pathological changes precipitated by smoking in these patients.

\section{Introduction}

It is a well known fact that environmental factors are as important as genetic factors in the carcinogenesis of urothelial cancer (UC). Cigarette smoking, in particular, is a major risk factor for cancer, with the risk of developing bladder cancer in smokers being several-fold that in non-smokers (1). In addition to carcinogenesis, cigarette smoking plays an important role in cancer progression and the prognosis of patients with bladder cancer (2-4). Although a similar role for smoking has been reported in patients with upper tract (UT) UC, there are conflicting opinions regarding the pathological significance and prognostic role of smoking status in these patients (5-9). In addition, it was previously suggested that the changes in the malignant potential of UTUC induced by cigarette smoke were different from those in bladder cancer (4). Therefore, a detailed investigation on the effect of smoking is crucial for understanding the fundamental characteristics of UTUC. However, the association between smoking and changes in pathological characteristics and molecular expression of cancer-related molecules in UTUC has not been fully elucidated. Cigarette smoke affects numerous pathological characteristics and molecules in vivo and in vitro. In the field of oncology, exposure to cigarette smoke may modify various cancer-related factors and molecules (10). In this study, we focused specifically on the following invasion- and metastasis-related factors: cancer cell proliferation, apoptosis, angiogenesis, lymphangiogenesis and expression of cyclooxygenase (COX)-2, vascular endothelial growth factors (VEGFs), matrix metalloproteinases (MMPs) and urokinase-type plasminogen activator (uPA), as these factors play important roles in UC (2,11-15).

The effect of smoking on the carcinogenesis, pathological characteristics and prognosis of UTUC has been reported and reviewed in earlier studies (5-9). Therefore, the main objective of this study was to elucidate the association between the smoking status of patients and the malignant aggressiveness 
of UTUC at the molecular level. Tumor growth, invasion and metastasis affect the quality of life and survival of patients with UTUC. Therefore, a detailed understanding of cancer cell dissemination and recurrence are crucial for the determination of appropriate observation and treatment strategies. Our results may provide new information on smoking-induced changes in the expression of cancer-related factors in patients with UTUC.

\section{Materials and methods}

Patients and tissue samples. A total of 134 patients diagnosed with non-metastatic UTUC were retrospectively investigated. Patients who had received preoperative therapy and/or who had carcinoma in situ (Cis) were excluded, due to difficulties in the evaluation of cancer-related factors and semi-quantification of immunological stainings. All the histological characteristics, including tumor grade and pT stage, were determined using formalin-fixed and paraffin-embedded specimens from open nephroureterectomy; cancer stage and grade were assessed using the 2002 tumor-node-metastasis (TNM) classification (16). The cancer was classified into three grades, namely G1, G2 and G3, according to the World Health Organization classification (17). A single pathologist performed all the pathological examinations. The median duration of the follow-up was 52 months (range, 2-250 months). The study protocol was approved by the Human Ethics Review Committee of the Nagasaki University Hospital.

Immunohistochemistry and evaluation of cancer-related factors. Immunohistochemical staining was performed according to our previous reports $(11,14,18-21)$. Briefly, $5-\mu \mathrm{m}$ sections were deparaffinized stepwise in xylene and rehydrated in graded solutions of ethanol. Antigen retrieval was performed at $121^{\circ} \mathrm{C}$ for $15 \mathrm{~min}$ (for the anti-Ki-67 antibody) or $95^{\circ} \mathrm{C}$ for $40 \mathrm{~min}$ (for all the other antibodies) in $0.01 \mathrm{M}$ sodium citrate buffer ( $\mathrm{pH}$ 6.0). All the sections were then immersed in $3 \%$ hydrogen peroxide for 30 min to block the endogenous peroxidase activity. The primary anti-Ki-67 and anti-D2-40 antibodies were obtained from Dako (Glostrup, Denmark); the anti-CD105 antibody was obtained from Vector Laboratories (Burlingame, CA, USA); the anti-VEGF-A antibody was obtained from Santa Cruz Biotechnology, Inc. (Santa Cruz, CA, USA); the anti-COX-2 antibody was obtained from Immuno-Biological Laboratories Co., Ltd. (Gunma, Japan); the anti-VEGF-C and anti-VEGF-D antibodies were obtained from Zymed Laboratories, Inc. (San Francisco, CA, USA); the anti-MMP-2 and anti-MMP-9 antibodies were obtained from Daiichi Fine Chemical Co., Ltd. (Toyama, Japan); and the anti-uPA antibody was obtained from American Diagnostica, Inc. (Stamford, CT, USA). The sections were incubated overnight at $4^{\circ} \mathrm{C}$ with the primary antibodies. Subsequently, the sections were treated with DAKO EnVision ${ }^{\mathrm{TM}}+$ Peroxidase (Dako, Carpinteria, CA, USA) for $60 \mathrm{~min}$ using the labeled polymer method. The peroxidase reaction was visualized using Liquid DAB Substrate kit (Zymed Laboratories, Inc). The sections were counterstained with hematoxylin, dehydrated stepwise through a graded alcohol series and cleared in xylene prior to mounting. Consecutive sections from each sample processed without the primary antibody were used as the negative control. In addition, in situ labeling for apoptosis was performed as previously described (18), using the ApopTag ${ }^{\circledR}$ In Situ Apoptosis Detection kit (Intergen Company, Purchase, NY, USA), which is based on the terminal deoxynucleotidyl transferase-mediated nick end labeling method.

Evaluation. All the analyses of immunohistochemically stained sections were performed using light microscopy within a tumor area containing $\geq 500$ carcinoma cells. In this study, lymphatic vessel density (LVD) was described in the peritumoral area according to previous report (20). Two investigators (S.W. and Y.M.), who were blinded to clinical data, independently performed the semi-quantitative analyses and the immunostaining interpretations. The disagreement rate for analyses between the two investigators was $<10 \%$ and results from both investigators were averaged for the statistical analyses. The presence of intratumoral lymphatic vessel (iLV) was defined as at least one D2-40 positive vessel clearly visible in the intratumoral area (20). As regards the expression of VEGF-A and -C, staining intensity was classified into four grades as follows: absent, weak, moderate and strong; the specimens were considered positive when $>25 \%$ of the carcinoma cells were clearly (moderately or strongly) stained for statistical evaluation according to a previous report (11). The expression of MMP-2 and -9 was also evaluated as previously reported (14). Briefly, the staining intensity was graded as weak, moderate, or intense. In addition, the extent of positive staining in the tumor area was graded as focal $(\leq 10 \%)$, regional $(11-50 \%)$, or diffuse $(\geq 50 \%)$. Finally, the staining patterns of moderate and diffuse, intense and regional, or intense and diffuse were considered to be positive for MMPs. For the evaluation of uPA, staining intensity was scored as 1 (weak), 2 (moderate) or 3 (intense staining). In addition, the extent of staining was scored as 1,2 or $3(<10$, $10-50$ or $>50 \%$ of tumor area stained, respectively). These two scores were summed up to give each case a final score (immunoreactive score of 0-6) and uPA was considered as positive when the immunoreactive score was 3-6 according to a previous report (21). Tumor sections stained with each antibody were examined under a Nikon E-400 bright-field microscope (Nikon, Tokyo, Japan) and images were captured using a digital camera (DU100; Nikon) at x200 magnification. For the assessment of microvessel density (MVD) and LVD, 3-5 fields with the greatest density of positively stained vessels (hot spots) were evaluated. Values were defined as the number of positively stained vessels per high-power field, as estimated from a computer-aided image analysis (WinROOF version 6.4; Mitani, Fukui, Japan).

Statistical analyses. Normality was evaluated by normal distribution and histograms for each variable. The results are expressed as mean \pm standard deviation (SD), unless otherwise stated. A Student's t-test was performed for continuous variables. The Scheffé test was used for multiple comparisons of data. Crude and adjusted effects were estimated by logistic regression analysis and described as odds ratios (ORs) with 95\% confidence intervals, together with P-values. For the survival analyses, variables that achieved statistical significance $(\mathrm{P}<0.050)$ in the univariate analyses were subsequently entered into a multivariate analysis using the Cox proportional 
Table I. Association between clinicopathological characteristics and smoking status.

\begin{tabular}{|c|c|c|c|c|}
\hline \multirow[b]{2}{*}{ Characteristics } & \multirow{2}{*}{$\begin{array}{l}\text { Total patient } \\
\text { number } \\
(\mathrm{n}=134)\end{array}$} & \multicolumn{3}{|c|}{ Smoking status at diagnosis, $\mathrm{n}(\%)$} \\
\hline & & Never $(n=54)$ & Former $(n=46)$ & Current $(n=34)$ \\
\hline Mean age (SD) & & $67.9(11.8)$ & $70.1(8.6)$ & $66.8(10.8)$ \\
\hline \multicolumn{5}{|l|}{ Gender } \\
\hline Male & 102 & $29(28.4)$ & $40(39.2)$ & $33(32.4)$ \\
\hline Female & 32 & $25(78.1)$ & $6(18.8)$ & $1(3.1)$ \\
\hline P-value & & & $<0.001$ & \\
\hline \multicolumn{5}{|l|}{ Grade } \\
\hline 1 & 24 & $6(25.0)$ & $11(45.8)$ & $7(29.2)$ \\
\hline 2 & 62 & $24(38.8)$ & $19(30.6)$ & $19(30.6)$ \\
\hline 3 & 48 & $24(50.0)$ & $16(33.3)$ & $8(16.7)$ \\
\hline P-value & & & 0.200 & \\
\hline \multicolumn{5}{|l|}{ pT stage } \\
\hline $\mathrm{Ta}$ & 13 & $6(46.2)$ & $4(30.7)$ & $3(23.1)$ \\
\hline $\mathrm{T} 1$ & 43 & $18(41.9)$ & $15(34.9)$ & $10(23.2)$ \\
\hline $\mathrm{T} 2$ & 24 & $7(29.2)$ & $9(37.5)$ & $8(33.3)$ \\
\hline $\mathrm{T} 3$ & 44 & $18(40.9)$ & $17(38.6)$ & $9(20.5)$ \\
\hline $\mathrm{T} 4$ & 10 & $5(50.0)$ & $1(10.0)$ & $4(40.0)$ \\
\hline P-value & & & 0.740 & \\
\hline Low (pTa and T1) & 56 & $24(42.9)$ & $19(33.9)$ & $13(23.2)$ \\
\hline High (pT2-4) & 78 & $30(38.5)$ & $27(34.6)$ & $21(26.9)$ \\
\hline P-value & & & 0.845 & \\
\hline
\end{tabular}

$\mathrm{SD}$, standard deviation.

hazards model. All the statistical tests were two-sided; statistical significance was set at $\mathrm{P}<0.050$. All the statistical analyses were performed on a personal computer using the StatView program of the Windows statistical package, version 5.0 (Abacus Concepts, Berkeley, CA, USA).

\section{Results}

Smoking status and clinicopathological characteristics. Our study population included patients who had never smoked/non-smokers $(n=54,40.3 \%)$, former smokers $(n=46$, $34.3 \%)$ and current smokers $(n=34,25.4 \%)$. The associations between the clinicopathological characteristics of UTUC and the smoking status of the patients are shown in Table I. Age at diagnosis in former smokers tended to be higher compared to that in other groups; however, this difference was not statistically significant. The number of former or current smokers was significantly higher $(\mathrm{P}<0.001)$ among male (39.2 and $32.4 \%$, respectively) compared to that among female patients (18.8 and 3.1\%, respectively). However, the pathological characteristics of UTUC exhibited no significant differences according to smoking status.

Smoking status and cancer-related factors. The associations between the smoking status of the patients and the cancer-related factors are shown in Table II. The proliferation index (PI) as well as the apoptotic index appeared to be posi- tively correlated with smoking status; however, the correlation was not statistically significant. However, the mean/SD level of LVD in current smokers (40.9/12.9) was significantly higher $(\mathrm{P}=0.034)$ compared to that in patients who had never smoked (34.4/10.6). In addition, smoking status was correlated with the presence of $\operatorname{iLV}(\mathrm{P}=0.010)$ and the expression of VEGF-D $(\mathrm{P}=0.011)$, COX-2 $(\mathrm{P}=0.032)$ and MMP-9 $(\mathrm{P}=0.032)$. With respect to the expression of VEGF-A, VEGF-C and MMP-2, although the positively stained ratio in current smokers was higher compared to that in those who had never smoked or former smokers, the differences were not statistically significant. Similarly, smoking status did not appear to be associated with MVD or uPA expression.

We next investigated the independent correlation between the smoking status and the cancer-related factors using a multivariate analysis model that included pathological characteristics. As shown in Table III, former smoking was independently associated with $\operatorname{LVD}(\mathrm{OR}=1.04, \mathrm{P}=0.024)$, the presence of $\mathrm{iLV}(\mathrm{OR}=5.1, \mathrm{P}=0.027)$ and VEGF-D expression $(\mathrm{OR}=3.0, \mathrm{P}=0.043)$, but not with the expression of COX-2 or MMP-9. However, current smoking was independently associated with all these factors (Table III).

Survival analyses. The Kaplan-Meier survival curves demonstrated that the smoking status of a patient was a significant predictor of the recurrence of urinary tract cancer (log-rank $\mathrm{P}=0.045$; Fig. 1A) and subsequent metastasis (log-rank 
Table II. Association between cancer-related factors and smoking status.

\begin{tabular}{|c|c|c|c|c|}
\hline \multirow[b]{2}{*}{ Factors } & \multicolumn{3}{|c|}{ Smoking status } & \multirow[b]{2}{*}{ P-value } \\
\hline & $\begin{array}{l}\text { Never } \\
(\mathrm{n}=54)\end{array}$ & $\begin{array}{l}\text { Former } \\
(\mathrm{n}=46)\end{array}$ & $\begin{array}{l}\text { Current } \\
(\mathrm{n}=34)\end{array}$ & \\
\hline Proliferation index (mean/SD) & $20.1 / 10.5$ & $21.2 / 10.3$ & $22.7 / 11.7$ & NS \\
\hline Apoptotic index (mean/SD) & $1.5 / 0.7$ & $1.6 / 0.8$ & $1.7 / 0.9$ & NS \\
\hline Microvessel density (mean/SD) & $31.0 / 16.4$ & $27.0 / 13.2$ & $32.1 / 19.6$ & NS \\
\hline Lymphatic vessel density (mean/SD) & $34.4 / 10.6$ & $39.6 / 10.8$ & $40.9 / 12.9$ & $0.034^{\mathrm{a}}$ \\
\hline Intratumoral lymph vessels, n (\%) & & & & 0.010 \\
\hline Absent & $51(94.4)$ & $37(80.4)$ & $24(70.5)$ & \\
\hline Present & $3(5.6)$ & $9(19.6)$ & $10(29.5)$ & \\
\hline VEGF-A expression, n (\%) & & & & 0.542 \\
\hline Negative & $30(55.6)$ & $22(47.8)$ & $15(44.1)$ & \\
\hline Positive & $24(44.4)$ & $24(52.2)$ & $19(55.9)$ & \\
\hline VEGF-C expression, n (\%) & & & & 0.779 \\
\hline Negative & $29(53.7)$ & $22(47.8)$ & $16(47.1)$ & \\
\hline Positive & $25(46.3)$ & $24(52.2)$ & $18(52.9)$ & \\
\hline VEGF-D expression, n (\%) & & & & 0.011 \\
\hline Negative & $47(47.0)$ & $33(33.0)$ & $20(20.0)$ & \\
\hline Positive & $7(20.6)$ & $13(38.2)$ & $14(41.2)$ & \\
\hline COX-2 expression, $\mathrm{n}(\%)$ & & & & 0.032 \\
\hline Negative & $34(63.0)$ & $21(45.7)$ & $12(35.3)$ & \\
\hline Positive & $20(37.0)$ & $25(54.3)$ & $22(64.7)$ & \\
\hline MMP-2 expression, $\mathrm{n}(\%)$ & & & & 0.359 \\
\hline Negative & $33(61.1)$ & $23(50.0)$ & $16(47.1)$ & \\
\hline Positive & $21(38.9)$ & $23(50.0)$ & $18(52.9)$ & \\
\hline MMP-9 expression, n (\%) & & & & 0.032 \\
\hline Negative & $35(64.8)$ & $21(45.7)$ & $13(38.2)$ & \\
\hline Positive & $19(35.2)$ & $25(54.3)$ & $21(61.8)$ & \\
\hline uPA expression, n (\%) & & & & 0.995 \\
\hline Negative & $24(44.4)$ & $20(43.5)$ & $15(44.1)$ & \\
\hline Positive & $30(55.6)$ & $26(56.5)$ & $19(55.9)$ & \\
\hline
\end{tabular}

${ }^{a}$ Never vs. current group. SD, standard deviation; NS, not significant; VEGF, vascular endothelial growth factor; COX, cyclooxygenase; MMP, matrix metalloproteinase; uPA, urokinase-type plasminogen activator.

$\mathrm{P}=0.016$; Fig. 1B), but not of cause-specific survival (log-rank $\mathrm{P}=0.514)$. Based on these results, the roles of various cancer-related factors in the recurrence of urinary tract carcinoma and subsequent metastasis were evaluated and are shown in Table IV. The Cox proportional hazard analyses indicated that the presence of iLV [hazard ratio $(\mathrm{HR})=2.5, \mathrm{P}=0.007$ ] and the expression of $\mathrm{COX}-2(\mathrm{HR}=2.1, \mathrm{P}=0.018)$ and MMP-9 ( $\mathrm{HR}=2.0, \mathrm{P}=0.026)$, but not LVD, were significantly associated with the recurrence of urinary tract cancer. However, similar analyses revealed that they were all significantly associated with subsequent metastasis (Table IV).

\section{Discussion}

The present study demonstrated that smoking leads to lymphangiogenesis and increased expression levels of COX-2 and
MMP-9 in patients with UTUC. Furthermore, these changes may play important roles in the recurrence of cancer in the urinary tract and in subsequent metastasis following nephroureterectomy, as these two molecules have been reported to be associated with recurrence and prognosis in patients with UC $(14,22,23)$. Although smoking is a major causative risk factor and a significant predictor of outcome in patients with UC, including UTUC $(5,9)$, the smoking-induced changes in the malignant behavior and expression of cancer-related factors have not yet been fully elucidated. To the best of our knowledge, this is the first study to describe the association between smoking status and the aggressiveness of cancer at the molecular level in human UTUC tissues.

The expression levels of VEGF-D, COX-2 and MMP-9, which are invasion- and metastasis-related molecules, were found to be significantly associated with smoking status in 
Table III. Correlation of different factors with smoking by multivariate analysis ${ }^{\mathrm{a}}$.

\begin{tabular}{lrcc}
\hline Factors & OR & $95 \%$ CI & P-value \\
\hline In former smokers & & & \\
LV density & 1.04 & $1.01-1.1$ & 0.024 \\
Presence of iLV & 5.1 & $1.2-21.7$ & 0.027 \\
VEGF-D: positive & 3.0 & $1.03-8.4$ & 0.043 \\
COX-2: positive & 2.2 & $0.9-5.1$ & 0.079 \\
MMP-9: positive & 2.0 & $0.9-4.7$ & 0.112 \\
In current smokers & & & \\
LV density & 1.1 & $1.01-1.1$ & 0.011 \\
Presence of iLV & 9.7 & $2.2-42.3$ & 0.003 \\
VEGF-D: positive & 5.3 & $1.8-15.5$ & 0.003 \\
COX-2: positive & 3.3 & $1.3-8.7$ & 0.014 \\
MMP-9: positive & 3.5 & $1.3-9.0$ & 0.011 \\
\hline
\end{tabular}

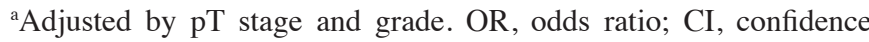
interval; iLV, intratumoral lymphatic vessels; COX, cyclooxygenase; MMP, matrix metalloproteinase.

\section{A}
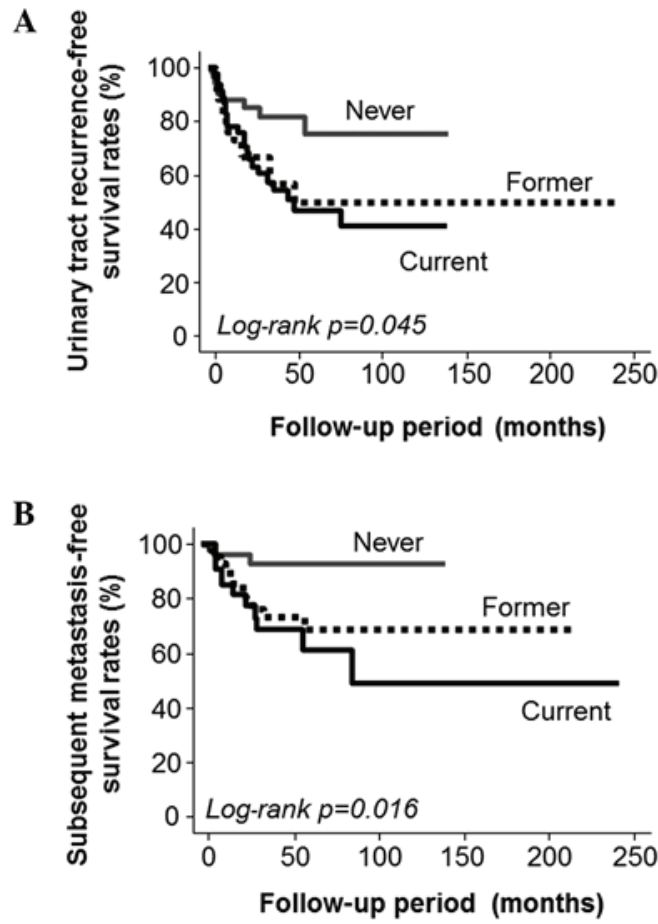

Figure 1. Kaplan-Meier curves of (A) urinary tract recurrence and (B) subsequent metastasis demonstrating that smoking status at diagnosis was significantly associated with urinary tract recurrence-free survival (log-rank $\mathrm{P}=0.045$ ) as well as subsequent metastasis-free survival following surgery ( $\log$-rank $\mathrm{P}=0.016)$.

our study population. In bladder cancer tissues, a recent study reported that VEGF-D overexpression was associated with a high frequency of lymph node metastasis and poor survival in bladder cancer (24). Interestingly, this study also demonstrated that VEGF-C expression did not play such significant pathological and prognostic roles. We hypothesized that this
Table IV. Cox proportional hazard analyses of smoking-related factors.

\begin{tabular}{|c|c|c|c|c|}
\hline \multirow[b]{2}{*}{ Factors } & \multirow[b]{2}{*}{ LVD } & \multirow{2}{*}{$\begin{array}{l}\text { Presence } \\
\text { of iLV }\end{array}$} & \multicolumn{2}{|c|}{ Positive expression } \\
\hline & & & $\mathrm{COX}-2$ & MMP-9 \\
\hline \multicolumn{5}{|c|}{$\begin{array}{l}\text { For urinary tract } \\
\text { recurrence }\end{array}$} \\
\hline Hazard ratio & 1.02 & 2.5 & 2.1 & 2.0 \\
\hline $95 \% \mathrm{CI}$ & $0.99-1.05$ & $1.3-4.8$ & $1.1-3.9$ & $1.1-3.6$ \\
\hline P-value & 0.087 & 0.007 & 0.018 & 0.026 \\
\hline \multicolumn{5}{|l|}{$\begin{array}{l}\text { For subsequent } \\
\text { metastasis }\end{array}$} \\
\hline Hazard ratio & 1.05 & 5.6 & 8.3 & 3.5 \\
\hline $95 \% \mathrm{CI}$ & $1.01-1.1$ & $2.5-12.3$ & $2.5-27.9$ & $1.5-8.4$ \\
\hline P-value & 0.002 & $<0.001$ & $<0.001$ & 0.005 \\
\hline
\end{tabular}

LVD, lymphatic vessel density; iLV, intratumoral lymphatic vessels; COX, cyclooxygenase; MMP, matrix metalloproteinase; CI, confidence interval.

phenomenon was the case not only in bladder cancer, but also in UTUC. There is little information on the association between COX-2 expression and UTUC according to smoking status. However, in an in vitro study using bladder cancer cell lines, cigarette smoke extract induced COX-2 expression in a time-dependent manner (25). Similar findings were also reported in human bladder cancer tissues $(3,26)$. Thus, various studies suggested that smoking mediates bladder cancer aggressiveness by regulating the expression of COX-2 (2). Based on these previously published reports and the results of the present study, we suggest that a similar positive correlation may exist in patients with UTUC and that it may represent one of the important mechanisms underlying the smoking-related malignant behavior of cancer cells. It is known that smoking induces polymorphism and expression of MMP-9 in bladder cancer (27); however, there is no report on the effect of smoking on MMP-9 expression in human UTUC tissues. In other types of cancer, cigarette smoke extract induces MMP-9 expression in vitro $(28,29)$. Of note, exposure to cigarette smoke also increases the expression levels of COX-2 in lung cancer tissue (28). Furthermore, in patients with cardiovascular diseases, several investigators have observed that smoking increases the expression level of MMP-9, but not that of MMP-2 $(30,31)$. Therefore, we hypothesized that smoking may stimulate cancer cell invasion and metastasis by inducing an increase in the expression levels of COX-2 and MMP-9 in patients with UTUC.

Several studies previously reported that the upregulation of lymphangiogenesis and lymphovascular invasion are crucial steps in cancer cell dissemination in patients with UTUC $(20,32)$. However, there is little information regarding the effect of smoking status on lymphangiogenesis in patients with UTUC. In patients with lung cancer, smoking was found to be associated with high expression levels of interleukin-17 and was hypothesized to play an important role in metastasis 
by promoting lymphangiogenesis (33). In addition, although the association between lymphangiogenesis and the expression of COX-2 or MMP-9 in UTUC is not fully understood, these two molecules have been reported to stimulate lymphangiogenesis in other types of cancer $(34,35)$. On the basis of these facts, we hypothesized that smoking induces cancer cell dissemination by stimulating lymphangiogenesis via the upregulation of COX-2 and MMP-9 expression in patients with urothelial carcinoma of the upper urinary tract (UC-UUT).

Our results demonstrated that the smoking status of patients exhibited no significant correlation with either cell proliferation or apoptosis. Similarly, smoking status was reported to have no significant correlation with PI in bladder cancer (36). However, a previous study suggested that smoking status is significantly associated with apoptosis in patients with bladder cancer (37). By contrast, other studies reported that the expression of apoptosis-related molecules, such as p53 and caspase-3, were not associated with smoking intensity in several cancers, including UC $(3,38)$. We suggest that the effect of smoking on cancer cell proliferation and apoptosis in patients with UC-UUT is limited.

VEGF-A is one of most potent pro-angiogenic molecules and its expression is known to be closely associated with MVD in several types of solid tumors. In addition, VEGF-A expression is a useful predictive factor of prognosis in patients with UC. Therefore, we hypothesized that, among UTUC patients, VEGF-A expression in current smokers may be significantly higher compared to that in non-smokers. However, various studies have demonstrated that VEGF-A is not invariably associated with smoking in UC. In fact, in an earlier study on bladder cancer, it was observed that cancer patients who smoked exhibited higher expression levels of VEGF-A compared to non-smokers; however, the difference between the two levels was not statistically significant (39). Similarly, several studies reported that there is no significant association between VEGF-A expression and smoking intensity in bladder cancer patients $(3,37)$. In addition, VEGF-A expression was not affected by the smoking status of patients with various disorders, including other smoking-related pathological conditions $(38,40)$. Therefore, we suggest that there may be no correlation between smoking and VEGF-A expression in patients with UTUC. In addition to VEGF-A, smoking has been reported to increase the activity and expression of VEGF-C under several pathological conditions, including cancer $(41,42)$. We previously reported that lymphangiogenesis is a crucial process in cancer cell dissemination in patients with UTUC (20). Additionally, the present study demonstrated that smoking stimulated lymphangiogenesis in UC-UUT patients. Therefore, we hypothesized that VEGF-C expression was significantly associated with the smoking status. However, such an association was not found in our study population.

Our results demonstrated that the smoking status of the patients was not associated with any of the pathological characteristics of UTUC, including muscle invasion and cancer grade. Initially, we hypothesized that the smoking status was closely associated with malignant potential in patients with UTUC. Indeed, we had previously reported that lymphangiogenesis and the expression of COX-2 and MMP-9 significantly affected malignant potential and tumor aggressiveness in patients with UTUC $(14,20)$. Furthermore, other previous reports demonstrated that the smoking status of these patients was significantly associated with pT stage and grade (6-8). However, there are conflicting opinions regarding these associations. For example, several studies reported that current smokers were significantly more likely to have a low pT stage and a low-grade cancer compared to non-smokers and former smokers $(6,7)$. Conversely, a previous study suggested that former and current smokers exhibit a higher pT stage and grade compared to non-smokers (8).

In addition to the correlation with pathological characteristics, although we observed that a higher intensity of smoking was a worse prognostic factor for cancer recurrence and metastasis following radical surgery, it was not found to be significantly associated with cause-specific survival in our study population. Several studies demonstrated that smoking intensity is a significant predictor for prognosis and survival in patients with UTUC $(6,7)$. However, a recent review demonstrated that there is a lack of consensus regarding the association between smoking status and cancer outcome, including cancer recurrence, progression and survival, in UTUC patients following nephroureterectomy (9).

Thus, there is no general agreement regarding the pathological significance and the prognostic role of smoking in patients with UTUC. Several investigators have suggested that this discrepancy may be attributed to the differences in study methodology, including the study population, and in the definition of high pT stage and grade. For example, in this study, to ensure the accurate evaluation of cancer-related factors and molecules, we excluded patients with Cis. In addition, we excluded patients with lymph node metastasis in order to accurately determine the association between smoking status and cancer-related factors.

In previous studies on the pathological role of smoking status, high pT stage was defined as pT2-4 (7) or pT3/4 (6) and tumor grade was divided into three (G1, G2 and G3) (7) or two (low and high) groups $(6,8)$. In addition, differences in smoking intensity and length of time after smoking cessation may result in such a discrepancy (8). Therefore, the differences in methodology, the diversity in patients' background, reliability of immunohistochemistry and accuracy of smoking status determination are potential limitations of such studies, including the present study. Furthermore, the effects of smoking on the malignant behavior and outcome of UTUC are complex. A previous study demonstrated that the recurrence-free survival rate was lower in current smokers compared to that in non-smokers (7), while a different study reported that smoking did not increase the risk of recurrence (6).

We were unable to explain the reasons why smoking status was associated with recurrence and metastasis following radical surgery, despite the fact that it was not associated with pathological characteristics at diagnosis. Therefore, detailed studies on the changes of pathological characteristics and on the effects of smoking at the molecular level are essential for a more accurate understanding of the effect of smoking on patients with UTUC. Although the small number of patients was a limitation of the present study, we emphasize that our findings may provide important information for urologists and medical oncologists.

In conclusion, our results demonstrated that the smoking status of the patients was associated with a recurrence 
of cancer in the urinary tract and subsequent metastasis following nephroureterectomy in patients with UC-UUT. Smoking-induced lymphangiogenesis and expression of COX-2 and MMP-9 appeared to be associated with such a prognosis. In addition to being carcinogenic and responsible for the stimulation of cancer-related factors, smoking is well known to be associated with other disadvantages, such as poor nutritional status and immune dysfunction. A complex mechanism appears to be associated with the smoking-related malignant behavior of cancer cells in UC-UUT. Therefore, larger and more detailed studies are required to ascertain appropriate observation and treatment strategies on the basis of the smoking status of patients with UC-UUT.

\section{Acknowledgements}

This study was supported in part by a Grant-in-Aid from the Japan Society for the Promotion of Science (to Yasuyoshi Miyata).

\section{References}

1. Strope SA and Montie JE: The causal role of cigarette smoking in bladder cancer initiation and progression, and the role of urologists in smoking cessation. J Urol 180: 31-37, 2008.

2. Huang RY and Chen GG: Cigarette smoking, cyclooxygenase-2 pathway and cancer. Biochim Biophys Acta 1815: 158-169, 2011.

3. Mitra AP, Castelao JE, Hawes D, et al: Combination of molecular alternations and smoking intensity predicts bladder cancer outcome: a report from the Los Angeles Cancer Surveillance Program. Cancer 119: 756-765, 2013.

4. Wang YH, Yeh SD, Wu MM, et al: Comparing the joint effect of arsenic exposure, cigarette smoking and risk genotypes of vascular endothelial growth factor on upper urinary tract urothelial carcinoma and bladder cancer. J Hazard Mater 262: 1139-1146, 2013.

5. Jensen OM, Knudsen JB, McLaughlin JK and Sørensen BL: The Copenhagen case-control study of renal pelvis and ureter cancer: role of smoking and occupational exposures. Int J Cancer 41: $557-561,1988$

6. Ehdaie B, Furberg H, Zabor EC, et al: Impact of smoking status at diagnosis on disease recurrence and death in upper tract urothelial carcinoma. BJU Int 111: 589-595, 2013.

7. Hagiwara M, Kikuchi E, Tanaka N, et al: Impact of smoking status on bladder tumor recurrence after radical nephroureterectomy for upper tract urothelial carcinoma. J Urol 189: 2062-2068, 2013.

8. Rink M, Xylinas E, Margulis V, et al: Impact of smoking on oncologic outcomes of upper tract urothelial carcinoma after radical nephroureterectomy. Eur Urol 63: 1082-1090, 2013.

9. Crivelli JJ, Xylians E, Kluth LA, Rieken M, Rink M and Shariat SF: Effect of smoking on outcomes of urothelial carcinoma: a systematic review of the literature. Eur Urol 65 : 742-754, 2014

10. Chen RJ, Chang LW, Lin P and Wang YJ: Epigenetic effects and molecular mechanisms of tumorigenesis induced by cigarette smoke: an overview. J Oncol 2011: 654931, 2011.

11. Miyata Y, Kanda S, Ohba K, et al: Lymphangiogenesis and angiogenesis in bladder cancer: prognostic implications and regulation by vascular endothelial growth factors-A, $-C$, and -D. Clin Cancer Res 12: 800-806, 2006.

12. Bolenz C and Lotan Y: Translational research in bladder cancer: from molecular pathogenesis to useful tissue biomarkers. Cancer Biol Ther 10: 407-415, 2010.

13. Birrane G, Li H, Yang S, Tachado SD and Seng S: Cigarette smoke induces nuclear translocation of heme oxygenase 1 (HO-1) in prostate cancer cells: Nuclear HO-1 promotes vascular endothelial growth factor secretion. Int J Oncol 42: 1919-1928, 2013.

14. Miyata Y, Kanda S, Nomata K, Hayashida Y and Kanetake H: Expression of metalloproteinase-2, metalloproteinase-9 and tissue inhibitor of metalloproteinase-1 in transitional cell carcinoma of the upper urinary tract: correlation with tumor stage and survival. Urology 63: 602-608, 2004.
15. Bhuvarahamurthy V, Schroeder J, Denkert C, et al: In situ gene expression of urokinase-type plasminogen activator and its receptor in transitional cell carcinoma of the human bladder. Oncol Rep 12: 909-913, 2004.

16. AJCC. Renal pelvis and ureter. In: American Joint Committee on Cancer. AJCC Cancer Staging Manual, 6th ed. New York, NY: Springer, pp329-334, 2002.

17. Mostofi FK, Sobin LH and Torloni H: Histological typing of urinary bladder tumors. In: International Histological Classification of Tumors, No. 10. Geneva: World Health Organization, 1973.

18. Miyata Y, Kanda S, Sakai H, Hakariya T and Kanetake H: Relationship between changes in prostate cancer cell proliferation, apoptotic index, and expression of apoptosis-related proteins by neoadjuvant hormonal therapy and duration of such treatment. Urology 65: 1238-1243, 2005.

19. Miyata Y, Sagara Y, Watanabe S, et al: CD105 is a more appropriate marker for evaluating angiogenesis in urothelial cancer of the upper urinary tract than CD31 or CD34. Virchows Arch 463: 673-679, 2013.

20. Miyata Y, Kanda S, Ohba K, et al: Tumor lymphangiogenesis in transitional cell carcinoma of the upper urinary tract: association with clinicopathological features and prognosis. J Urol 176: 348-353, 2006.

21. Ohba K, Miyata Y, Kanda S, Koga S, Hayashi T and Kanetake H: Expression of urokinase-type plasminogen activator, urokinase-type plasminogen activator receptor and plasminogen activator inhibitors in patients with renal cell carcinoma: correlation with tumor associated macrophage and prognosis. J Urol 174: 461-465, 2005.

22. Oku S, Higashi M, Imazono Y, et al: Overexpression of cyclooxygenase-2 in high-grade human transitional cell carcinoma of the upper urinary tract. BJU Int 91: 109-114, 2003.

23. Ishida M, Mikami S, Kikuchi E, et al: Activation of the aryl hydrocarbon receptor pathway enhances cancer cell invasion by upregulating the MMP expression and is associated with poor prognosis in upper urinary tract urothelial cancer. Carcinogenesis 31: 287-295, 2010.

24. von Hardenberg J, Martini T, Knauer A, et al: Expression and predictive value of lymph-specific markers in urothelial carcinoma of the bladder. Urol Oncol 32: 54.e9-17, 2014.

25. Kamat AM, Sethi G and Aggarwal BB: Curcumin potentiates the apoptotic effects of chemotherapeutic agents and cytokines though down-regulation of nuclear factor-kappaB and nuclear factor-kappaB-regulated gene products in IFN-alpha-sensitive and IFN-alpha-resistant human bladder cancer cells. Mol Cancer Ther 6: 1022-1030, 2007.

26. Badawi AF, Habib SL, Mohammed MA, Abadi AA and Michael MS: Influence of cigarette smoking on prostaglandin synthesis and cyclooxygenase-2 gene expression in human urinary bladder cancer. Cancer Invest 20: 651-656, 2002.

27. Srivastava P, Mandhani A, Kapoor R and Mittal RD: Role of MMP-3 and MMP-9 and their haplotypes in risk of bladder cancer in North Indian cohort. Ann Surg Oncol 17: 3068-3075, 2010.

28. Shishodia S, Potdar P, Gairola CG and Aggarwal BB: Curcumin (diferuloylmethane) down-regulates cigarette smoke-induced NF-kappaB activation through inhibition of IkappaBalpha kinase in human lung epithelial cells: correlation with suppression of COX-2, MMP-9 and cyclin D1. Carcinogenesis 24: 1269-1279, 2003.

29. Ye YN, Wu WK, Shin VY and Cho CH: A mechanistic study of colon cancer growth promoted by cigarette smoke extract. Eur J Pharmacol 519: 52-57, 2005.

30. Dellalibera-Joviliano R, Jacob-Ferreira AL, Joviliano EE, Tanus-Santos JE and Evora PR: Imbalanced matrix metalloproteinase- 9 and tissue inhibitor of metalloproteinase-1 activities in patients with thromboangiitis obliterans. Vasc Med 17: 73-78, 2012.

31. Sivaraman SK, Zachariah G and Annamala P: Effect of smoking on metalloproteinases (MMPs) activity in patients with acute myocardial infarction (AMI). J Clin Diagn Res 8: 27-30, 2014.

32. Ku JH, Byun SS, Jeong H, Kwak C, Kim HH and Lee SE: Lymphovascular invasion as a prognostic factor in the upper urinary tract urothelial carcinoma: a systematic review and meta-analysis. Eur J Cancer 49: 2665-2680, 2013.

33. Chen X, Wan J, Liu J, et al: Increased IL-17-producing cells correlate with poor survival and lymphangiogenesis in NSCLC patients. Lung Cancer 69: 348-354, 2010. 
34. Yoo YA, Kang MH, Lee HJ, et al: Sonic hedgehog pathway promotes metastasis and lymphangiogenesis via activation of Akt, EMT, and MMP-9 pathway in gastric cancer. Cancer Res 71: 7061-7070, 2011.

35. Morita Y, Hata K, Nakanishi M, Nishisho T, Yura Y and Yoneda T: Cyclooxygenase-2 promotes tumor lymphangiogenesis and lymph node metastasis in oral squamous cell carcinoma. Int J Oncol 41: 885-892, 2012

36. Lenz P, Pfeiffer R, Baris D, et al: Cell-cycle control in urothelia carcinoma: large-scale tissue array analysis of tumor tissue from Maine and Vermont. Cancer Epidemiol Biomarkers Prev 21: 1555-1564, 2012.

37. Rahmani A, Alzohairy M, Khadri H, Mandal AK and Rizvi MA: Expressional evaluation of vascular endothelial growth factor (VEGF) protein in urinary bladder carcinoma patients exposed to cigarette smoke. Int J Clin Exp Pathol 5: 195-202, 2012

38. Scheidt JH, Yurgel LS, Romanini J, Cherubini K, de Figueiredo MA and Salum FG: Oral squamous cell carcinoma from users and nonusers of tobacco and alcohol: clinicopathologic features and immunoreactivity of VEGF, caspase-3, and p53. Appl Immunohistochem Mol Morphol 21: 148-153, 2013.
39. Quentin T, Schlott T, Korabiowska M, et al: Alteration of the vascular endothelial growth factor and angiopoietins-1 and -2 pathways in transitional cell carcinomas of the urinary bladder associated with tumor progression. Anticancer Res 24: 2745-2756, 2004.

40. Mondul AM, Rager HC, Kopp W, Virtamo J and Albanes D: Supplementation with alpha-tocophenol or beta-carotene reduces serum concentrations of vascular endothelial growth factor-D, but not -A or -C, in male smokers. J Nutl 141: 2030-2034, 2011.

41. Lane D, Gray EA, Mathur RS and Mathur SP: Up-regulation of vascular endothelial growth factor-C by nicotine in cervical cancer cell lines. Am J Reprod Immunol 53: 153-158, 2005.

42. Inamine M, Nagai Y, Mitsuhashi A, et al: Cigarette smoke stimulates VEGF-C expression in cervical intraepithelial neoplasia (CIN) 1 and 2 lesions. Int J Clin Oncol 17: 498-504, 2012. 